\title{
Adaptive Extraction of Oil Painting Texture Features Based on Reaction Diffusion Equation
}

\author{
Qicai Huang \\ Department of Art and Design, Taiyuan University, Taiyuan, Shanxi Province 030032, China \\ Correspondence should be addressed to Qicai Huang; sxty2008@tyu.edu.cn
}

Received 14 September 2021; Accepted 4 October 2021; Published 3 November 2021

Academic Editor: Miaochao Chen

Copyright ( 2021 Qicai Huang. This is an open access article distributed under the Creative Commons Attribution License, which permits unrestricted use, distribution, and reproduction in any medium, provided the original work is properly cited.

\begin{abstract}
The oil painting retrieval technology based on the reaction diffusion equation has attracted widespread attention in the fields of oil painting processing and pattern recognition. The description and extraction of oil painting information and the classification method of oil paintings are two important processes in content-based oil painting retrieval. Inspired by the restoration and decomposition functional model of equal oil painting, we propose a reaction diffusion equation model. The new model contains two reaction diffusion equations with different principal parts. One principal part is total variation diffusion, which is used to remove noise. The other main part is thermal diffusion, which is used to modify the source term of the denoising reaction-diffusion equation to achieve the effect of protecting the texture of the oil painting. The interaction of the two reaction-diffusion equations finally achieves denoising while maintaining the boundaries and textures. Under the framework of the above reaction diffusion equation model, we introduce Laplace flow to replace the original total variation flow, so that the new denoising reaction diffusion equation combines the isotropic diffusion and total variation flow of the thermal reaction diffusion equation to achieve the effect of adaptive theoretical research. Using regularization methods and methods, we, respectively, get the well-posedness of the two model solutions, which provides the necessary preparation for numerical calculations. Based on the statistical theory and classification principles of support vector machines, combined with the characteristics of oil painting classification, the research and analysis are carried out from the three important aspects of kernel function, training algorithm, and multiclass classifier algorithm that affect the classification effect and speed. Numerical experiments show that the given filter model has a better processing effect on images with different types and different degrees of noise pollution. On this basis, an oil painting classification system based on texture features is designed, combined with an improved gray-level cooccurrence matrix algorithm and a multiclass support vector machine classification model, to extract, train, and classify oil paintings. Experiments with three types of oil paintings prove that the system can achieve a good oil painting classification effect. Different from the original model, the new model is based on the framework of reaction-diffusion equations. In addition, the new model has good effects in removing step effects, maintaining boundaries and denoising, especially in maintaining texture.
\end{abstract}

\section{Introduction}

Generally speaking, oil painting texture processing technology takes digital oil painting as the object, including oil painting texture acquisition, oil painting texture analysis, and oil painting texture understanding. Oil painting analysis is a link between oil painting acquisition and oil painting understanding, which directly affects the degree of computer comprehension [1]. The problems of oil painting restoration and oil painting segmentation belong to the research category of oil painting analysis and have always been two important and widely studied issues in oil painting processing [2]. With the increasing demand and breakthroughs in physical technology, people pay close attention to and explore the essence of oil painting processing and try to use strict mathematical theory to classify and improve the existing oil painting processing models [3]. Currently, digital oil painting processing technology has three main tools: random theory, wavelet analysis theory, and partial differential reaction diffusion equation theory. Among them, the stochastic model is directly applied to oil painting based on Bayesian estimation and Markov random field, and many 
reasonable models have been established, while wavelet analysis is based on Fourier analysis to transform oil painting information into the frequency domain and then build a model on the basis of it, which indirectly acts on oil painting texture recognition [4].

The noise and detailed texture of the oil painting are in the high-frequency area of the oil painting, and most of the information in the oil painting is stored in the edge part. In the process of removing the oil painting noise, the texture detail information of the oil painting will be mistakenly regarded as the noise information of the oil painting and be filtered out, resulting in the loss of image information and blurring of the oil painting [5]. Obtaining highresolution oil paintings often requires high-cost and complex systems. In order to obtain finer details of the imaging scene under given system parameters, image interpolation methods can be used [6]. The usual nearest neighbor interpolation method, bilinear interpolation method, bicubic interpolation method, cubic convolution interpolation method, etc. enlarge the original oil painting, which improves the visual effect of the oil painting to a certain extent, but the interpolation precision of these algorithms is not high enough. The visual effect of oil painting is not improved enough [7]. The model parameters and missing pixels are estimated through nonlinear optimization methods, and the model parameters and missing pixels are estimated at the same time using nonlinear optimization methods. Therefore, the protection of oil painting details and texture information and the removal of oil painting noise information have become an irreconcilable contradiction. The goal of oil painting denoising is to keep the edge and texture details as much as possible while removing noise [8]. However, traditional oil painting denoising algorithms cannot balance the above two contradictions. It is imperative to find an algorithm that can well balance the contradiction between noise elimination and edge preservation information. The oil painting denoising algorithm based on the reaction diffusion equation can selectively smooth the oil painting and better balance the contradiction between the two [9]. This paper uses a combination of theoretical analysis and simulation experiments, combined with fractional calculus and fidelity terms, to study the oil painting denoising model based on the reaction-diffusion equation.

By analyzing the dependence of the oil painting boundary on the position and structure of the oil painting, we construct a boundary mapping function with strong antinoise ability. The corresponding reaction diffusion equation not only has the characteristics of anisotropic diffusion but also conducts heat in the homogeneous area inside the oil painting. The average curvature diffusion is carried out in the near-boundary area, and finally, the effect of adaptive denoising is achieved. In terms of theoretical research, we first use the fixed-point method to prove the wellposedness of the new model, and then study the asymptotic state of the solution, and the conclusion shows that the new model limit state of the recovery result is the local mean value of the initial oil painting. Compared with the TV model, the new model conducts thermal diffusion in the internal homogeneous region, effectively avoiding the step effect. Compared with the PM model, the new model is well-posed, so the solution is more stable, and due to the diffusion mode and diffusion speed of the new model, the model has stronger adaptive ability, better denoising in the internal homogeneous area, and more precise maintenance of the border near the border. This paper studies a variety of texture features in the oil painting content description and extraction method. Several numerical experiments verify the robustness of the proposed index against changes in translation, scale, and rotation. The application of image denoising shows the effectiveness of the proposed index. Starting from the characteristics of texture and the practical application of oil painting content retrieval technology, the gray-level cooccurrence matrix algorithm in the statistical method is analyzed emphatically. Aiming at the shortcomings of this method that it has a large amount of redundant calculations and requires a large amount of storage space, several existing improved algorithms based on gray-level cooccurrence matrix algorithms are further studied, including the sum-and-difference statistical method algorithms.

\section{Related Work}

Many researchers have done further research along this route. Xing et al. [10] studied statistical information, and for the first time experimented and proposed that the deficit moment, contrast, and entropy have the greatest recognition ability. The two-dimensional cooccurrence matrix proposed by the predecessors discarded the color information, and Zhang et al. [11] proposed a three-dimensional form of the cooccurrence matrix to solve this problem. Based on the psychological research on the visual perception of texture by the human eye, Yao et al. [12] proposed six texture attributes that can constitute a texture visual model, which are contrast, granularity, directionality, line type, roughness, and uniformity. Zhang et al. [13] found that the gradient cooccurrence matrix method, which combines the boundary operator and the gray distribution, can better describe the texture characteristics. This method first convolves the five special convolution kernels in pairs and then convolves the oil painting on the template obtained from the convolution and extracts texture features. Chi et al. [14] proposed a two-stage self-supervised texture segmentation method. The method first obtains the initial segmentation map through unsupervised automatic regression clustering and then performs self-supervised wavelet classification on this basis. Wen et al. [15] introduced a statistical texture analysis method based on texture element mode, which can provide spatial structure information of oil painting texture and has the monotonic invariance of pixel gray value. Zhao et al. [16] proposed a texture analysis method based on BVLC moments and BDIP moments. BVLC can display rough and smooth characteristics, and BDIP can extract troughs and edges very well. They are processed directly on the color space, which can effectively combine color features. The frequency domain analysis method of texture has also been developed rapidly.

As a remedy, nonlinear technology has successfully coordinated the processing of oil painting denoising and edge 
enhancement and has become a very effective technical method. Among them, nonlinear diffusion technology has been a hot topic in recent years, including the reaction diffusion equation method and geometric method, among which good results have been achieved in oil painting enhancement, oil painting segmentation and edge detection, texture generation, and mathematical morphology. The high-quality results concerned are computer vision and pattern recognition [17]. The method based on the Gabor filter conforms to the characteristics of the human visual perception system and the physiological vision of the human eye and is an important development direction for texture oil painting analysis. Gabor wavelet is a joint space-frequency method, and its texture model is based on a narrow-band texture field model, which can achieve local optima in both the frequency domain and the spatial domain. After the theoretical structure of wavelet transform was established, many scholars began to study how to use wavelet transform to represent texture features. Some scholars use statistics (variance and average) extracted from wavelet subbands as texture features, which have good retrieval results [18]. It includes the use of wavelet frame transform package theory to analyze the texture of oil paintings to obtain the characteristics of translation invariance and stability. In order to explore the characteristics of medium waves, scholars use tree-shaped wavelet transform to further improve the accuracy of classification. The combination of wavelet transform and other technologies achieves better performance.

The model uses directional diffusion with protective edge characteristics instead of isotropic diffusion with Gaussian smooth kernel, laying the theoretical foundation of nonlinear diffusion method for oil painting processing, and opening up a new field of oil painting processing application research. Based on the same framework, the introduction of total variation further demonstrates the importance of PDE models and theories in oil painting processing. The use of nonlinear diffusion methods to obtain continuous models makes mesh selection and isotropic diffusion easier to deal with; nonlinear diffusion methods are also allowed to merge or separate known filtering methods and form new models; further PDE models make algorithm analysis and synthesis more natural and flexible to achieve high accuracy and stability [19-21]. Gaussian filtering, median filtering, and their improvements all have the advantage of a small amount of calculation. This is indispensable in the real-time processing of computer vision. For the need to restore high-quality oil paintings, nonlinear anisotropic diffusion model is an important method $[22,23]$.

\section{Construction of an Adaptive Extraction Model of Oil Painting Texture Features Based on Reaction-Diffusion Equations}

3.1. Distribution of the Solution Set of the Reaction-Diffusion Equation. There are two important frameworks for oil painting restoration and decomposition models based on reaction-diffusion equations. The variational model is based on energy functionals, and the reaction-diffusion equation model is based on fluid diffusion theory. First, we review the basic frameworks of denoising models based on variational methods and some classic models.

$$
\begin{aligned}
& f(x(n))=\{x(i)\}, i=1, \cdots, n ; n \in R, \\
& \alpha(i, j, k)=(i(1), i(2) ; j(1), j(2) ; k(1), k(2)), \quad i, j, k \in Z .
\end{aligned}
$$

The value on the main diagonal of the gray-level cooccurrence matrix is used to measure the smoothness of the texture. The closer the gray pair value is to the main diagonal value, the smoother the texture is. The eigenvalues DIS, CON, INV, and IDM reflect the relationship between the value of the gray pair and the value of the main diagonal, so they can express the smoothness of the texture. DIS and CON are inversely proportional to INV and IDM, respectively. The CON value measures the size of the texture change.

Figure 1 shows the spatial distribution of the solution set of the reaction diffusion equation. According to the known training samples, the characteristic parameters are selected, and the discriminant function is established to classify each object. This method must have prior knowledge of the classification area to establish the discriminant function and obtain the training classifier.

$$
\begin{gathered}
\sum_{i}|<\alpha(1), u(i)>|^{2}+\sum_{j}|<\alpha(2), \quad u(j)>|^{2}=|u(i, j)|^{2}, \\
\left\{\begin{array}{l}
u(x, y)=\bar{u}(x, y), \\
v(x, y)=\bar{v}(x, y),
\end{array} \quad x, y \in \bar{S}(\bar{u}) .\right.
\end{gathered}
$$

The supervised classification method mainly decomposes oil paintings or objects into several main parts and seeks the spatial relationship between the parts. Usually, the supervised classification method needs to manually select the classification area to determine the model in the training stage.

$$
\frac{\partial u}{\partial x}+\frac{\partial v}{\partial y}+\frac{\partial w}{\partial z}=0
$$

First, the original problem is divided into a linear subproblem and a nonlinear subproblem by the time splitting method. Then, for the linear subproblem, considering the outer part of the given artificial region, the one-way approximation operator of the linear differential operator can be obtained. Finally, the local absorbing boundary condition is obtained by combining the approximation operator and the nonlinear operator.

$$
\left\{\begin{array}{l}
\phi\left(i_{1}\right)=\frac{\phi^{+}(i)+\phi^{-}(i)}{2}, \\
\phi\left(i_{2}\right)=\frac{\phi^{+}(i)-\phi^{-}(i)}{2} .
\end{array}\right.
$$




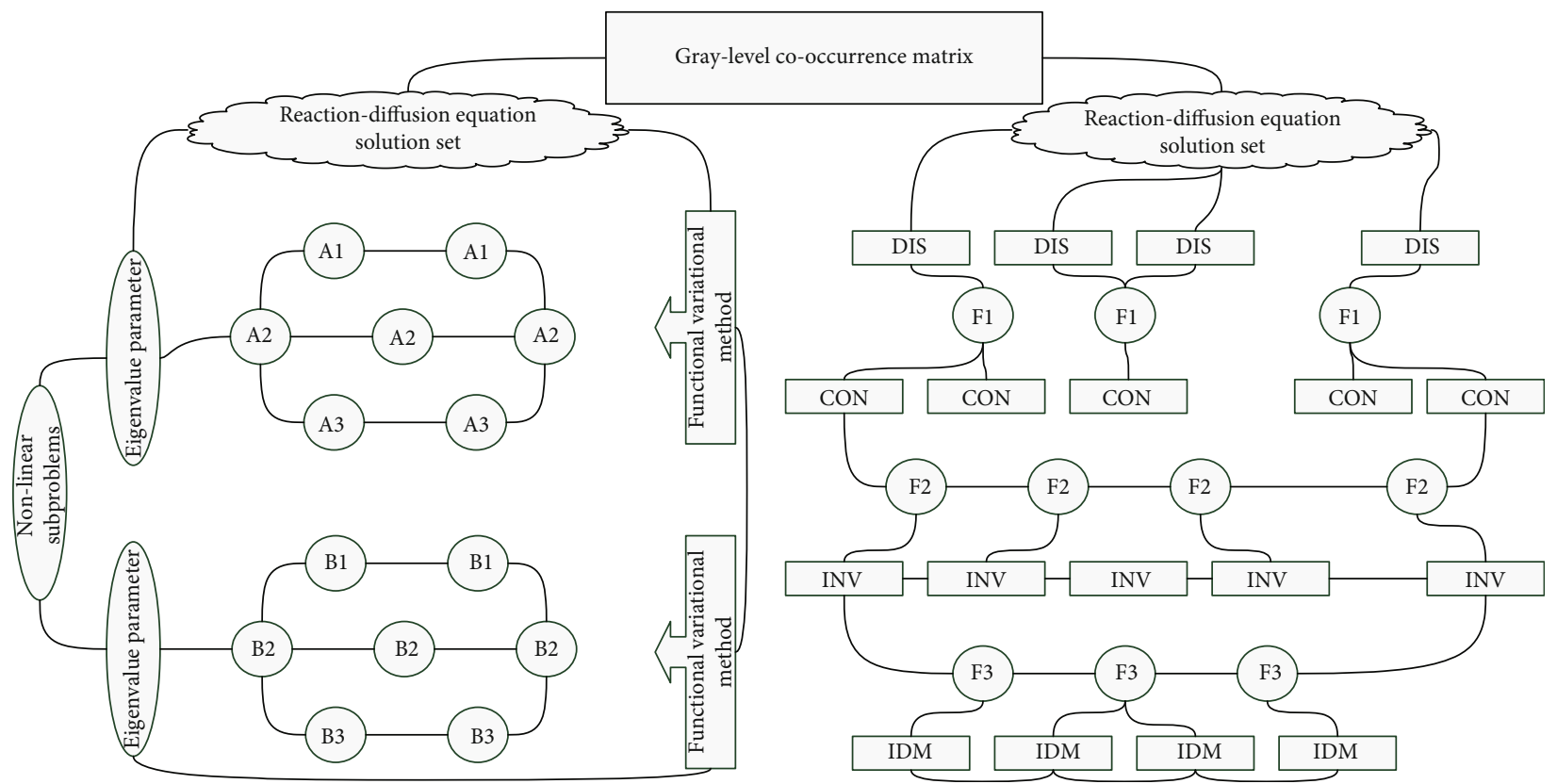

FIgURE 1: The spatial distribution of the solution set of the reaction-diffusion equation.

These grid-moving parameters in the reaction-diffusion equation have a time polishing effect. If the value is too small, the rigidity of the reaction diffusion equation obtained will be very large, which will bring difficulties to the numerical integration. If it is too large, the obtained mesh will not achieve the effect of equal distribution. That is to say, the solution of the reaction-diffusion equation will become infinite in a finite time. Since the singularity occurs in a very small space or time scale, it is very necessary to adopt an adaptive method in the numerical simulation.

$$
\sigma(x, y)=\frac{\varepsilon(x, x)}{E \cdot(\varepsilon(x, x)-t(\varepsilon(y, y)))}
$$

The method based on the grid position directly controls the position of the grid point. Most of the popular variational grid generation methods in the moving grid method belong to this category. The variational grid adaptive method first introduces a functional to control the density of the grid and often adds some properties of the grid, such as smoothness and regularity.

$$
\left\{\begin{array}{l}
\varepsilon(x, x)=\frac{\partial u}{\partial x} \\
\varepsilon(y, y)=\frac{\partial v}{\partial y} \\
\varepsilon(z, z)=\frac{\partial w}{\partial z}
\end{array}\right.
$$

The reaction-diffusion equation can be seen as a continuous reflection of pieces bounded by the edge of the painting. Under the control of the diffusion operator, the initial oil painting is diffused, and the model realizes selective diffu- sion smoothing according to the oil painting gradient model. Because the edge part has a larger gradient modulus, take a smaller value at this time, and the model implements weaker smoothing at the edge to protect the edge information: in a flat area, the gradient modulus is smaller, and the degree of diffusion becomes larger.

$$
\partial h(\partial x, \partial y, \partial z)=\left[\begin{array}{cccc}
h_{x x} & 0 & \cdots & h_{x y} \\
0 & h_{x x} & \cdots & 0 \\
\cdots & \cdots & \cdots & \cdots \\
h_{x y} & 0 & \cdots & h_{x x}
\end{array}\right] .
$$

The model automatically smooths less at the edge and much smoother at the flat. It can identify the boundary position to a certain extent, so it can better solve the contradiction between denoising and edge retention, and it can repair the disconnection due to noise pollution. In physics, the form of this reaction-diffusion equation is equivalent to the thermal reaction-diffusion equation. The parameter in it is also called the diffusion coefficient. Due to the diffusion coefficient, the diffusion speed in each direction changes with the gradient mode, which is different from the nondirectional ones. The isotropic reaction-diffusion equation is called the anisotropic reaction-diffusion equation.

$$
\begin{aligned}
& \min \left\{\operatorname{TV}(u)-\text { s.t. }|f-u(i, j)|_{t}^{2}\right\}=\sigma^{2}, \\
& \quad\{\sigma(x, x), \sigma(y, y), \sigma(z, z)\} \longrightarrow\{\varepsilon(x, y), \varepsilon(y, z), \varepsilon(z, x)\} .
\end{aligned}
$$

With an adaptive grid, the grid points will be dense to the singular area, so that the details of the singular area can be well-described. This effect will be more obvious when 
using numerical methods to solve differential equations. Especially for the time-dependent differential reactiondiffusion equations, the calculation errors in each layer of time will add up. In this case, if an inappropriate grid is used, the numerical solution may even produce oscillations. We often need to find a suitable grid for each time level. Therefore, when the singular area changes with time, our grid generally also changes with time.

3.2. Oil Painting Texture Feature Extraction Algorithm. In oil painting texture processing, oil painting texture restoration has always been one of the most important basic research topics. In the process of image imaging, copying, scanning, transmission, display, etc., it is inevitable to cause oil painting degradation, such as blur and noise interference. In many application fields, clear, high-quality oil paintings are required. Therefore, oil painting restoration, such as denoising and deblurring, has important meaning.

$$
\begin{gathered}
p(x, t)+\nabla \frac{\nabla u(x, t)^{k}}{\left|\nabla u(x, t)^{k}\right|}=0, \\
\varepsilon(x, x)=\frac{p^{\prime}(x)-p(x)}{p(x)}=u_{x} .
\end{gathered}
$$

Noise can be considered unpredictable in theory and subject to random errors of a certain probability distribution. For the convenience of analysis and processing, we assume that the oil painting signal and noise are independent of each other. The central task of oil painting restoration is to find the original ideal oil painting, and the second oil painting decomposition can be regarded as oil painting denoising when the statistical information of the degraded oil painting and noise is known. The extension of the denoising model can basically be used as a decomposition model of oil painting. Table 1 shows the distribution of oil painting texture characteristic attributes. With the development of oil painting processing technology, oil painting decomposition has begun to have some of its own characteristics. For this reason, we need to propose and analyze oil painting decomposition models from a new perspective.

Using signal processing methods, the spatial domain texture oil painting is transformed into the frequency domain mainly by Fourier transform; by calculating the area at the peak, the square of the distance between the peak and the origin is given. Difference and so on are studied to obtain texture features that are not easy to obtain in the spatial domain, such as period and power spectrum information.

$$
\begin{aligned}
& \widehat{s}=\frac{k(1) k(2)}{\lim _{k 1, k 2 \longrightarrow \infty} \sum_{i=0}^{k 1} \sum_{j=0}^{k 2} s(i, j) \times p(x, t)}, \\
& U=\frac{1}{2 \int_{\Omega} \sigma(z, x) \sigma(x, y) \sigma(y, z) d \Omega} .
\end{aligned}
$$

Generally, the protruding peaks in the Fourier spectrum correspond to the main direction information of the texture, the position of the peak in the frequency domain corre-
TABLE 1: Oil painting texture characteristic attribute distribution.

\begin{tabular}{lccc}
\hline $\begin{array}{l}\text { Texture } \\
\text { index }\end{array}$ & $\begin{array}{c}\text { Salt and pepper noise } \\
(\%)\end{array}$ & $\begin{array}{c}\text { Gaussian } \\
\text { noise }\end{array}$ & $\begin{array}{c}\text { Speckle noise } \\
(\%)\end{array}$ \\
\hline \multirow{2}{*}{ Group A } & 0.03 & 0.02 & 0.005 \\
& 0.01 & 0.03 & 0.01 \\
Group B & 0.04 & 0.04 & 0.01 \\
& 0.02 & 0.01 & 0.015 \\
\hline
\end{tabular}

sponds to the periodic information of the texture, the frequency components of the coarse texture are concentrated in the low-frequency part, and the frequency components corresponding to the fine texture are concentrated in the high-frequency part. The method of analyzing the shape and arrangement and distribution characteristics of the texture primitives of oil painting from the structural point of view is called the structural analysis method. The structural analysis method first determines the texture primitives based on the characteristics of eccentricity, area, direction, moment, extension, Euler number, amplitude, perimeter, etc. and then uses formal language to describe the texture arrangement rules based on the syntactic pattern recognition theory. The structural method is mainly used in the case of known primitives, using morphology, topology, graph theory, and other methods to describe the spatial geometric characteristics and arrangement rules of texture primitives.

Figure 2 shows the flow chart of oil painting texture feature extraction. Among the three basic features of oil painting, texture feature as a significant visual feature, not only does not depend on color or brightness but also includes the arrangement and organization order of the surface structure of things, showing the connection of context and content, reflecting the homogeneity of the oil painting. It is generally believed that the texture reflects the gray-scale repetition or change between oil painting pixels, as well as the repetition or change of color in space. The basic elements that make up a texture are called texture primitives. The texture primitive is defined as a basic unit with certain invariable characteristics that induces visual perception. These visual units reappear in different directions and with different deformations in different directions within a given area, which is expressed as an oil painting in gray. The visual unit usually contains multiple pixels, and the surface of the object can present three situations: periodicity, quasiperiodicity, or randomness. The signal can be analyzed in the time and frequency domain at the same time, and there is an automatic zoom function, that is; when the resolution in the frequency domain is high, the resolution in the time domain is low; when the resolution in the frequency domain is low, the resolution in the time domain is high. Generally, texture contains two basic elements: texture primitives and the arrangement rules of texture primitives. Among them, the arrangement rules of texture primitives may be random, or they may be regular.

3.3. Adaptive Optimization of Model Factors. Extracting texture features through a certain oil painting processing method has two main purposes: one is to detect the texture 


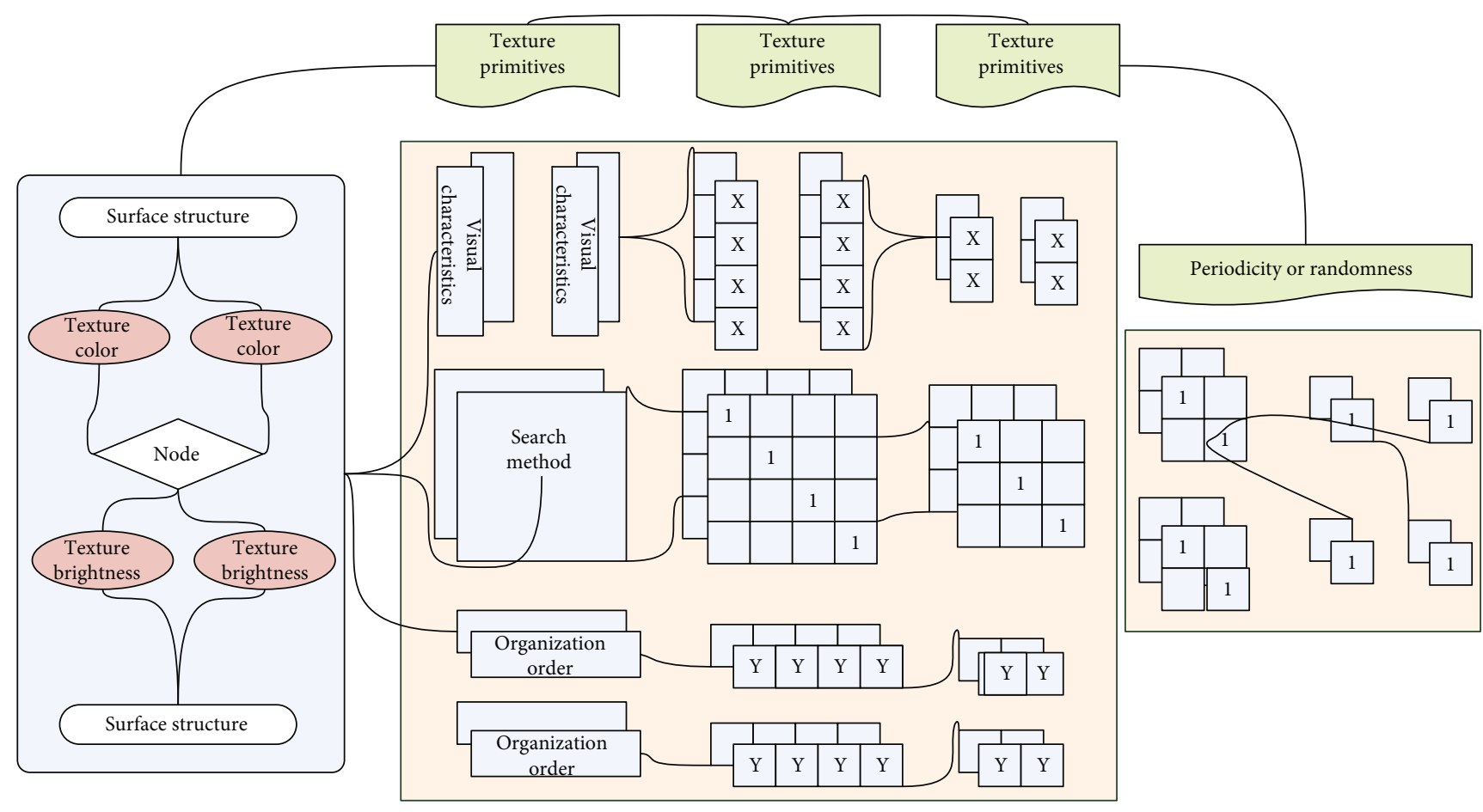

FIGURE 2: Flow chart of oil painting texture feature extraction.

primitives contained in the oil painting; the other is to obtain the characteristic information of the arrangement and distribution of these texture primitives. The description of oil painting texture usually uses the structural or statistical characteristics of the texture. The spatial-based nature of the texture can also be analyzed by converting it to the frequency domain. Therefore, there are three commonly used texture description methods: statistical method, structural method, and frequency spectrum method. Firstly, a onedimensional filter template is defined, and the texture attributes are determined by estimating the average gray level, ripples, waveforms, edges, and spots in the texture. After the one-dimensional filter templates are convolved with themselves, 5 vectors are generated. The first term is used as a column vector, and the second term is used as a row vector, resulting in a $5 \times 5$ law mask. The mask is convolved with the texture oil painting, and the calculated energy is used as a feature to describe the texture. Figure 3 shows the fit of the texture eigenvalues of different vectors.

The effectiveness of the template mainly depends on the local maximum of the template matching, not the specific number used in the template. After obtaining the graylevel cooccurrence matrix in the four directions, the following texture eigenvalues defined by the gray-level cooccurrence matrix algorithm need to be calculated, respectively, and finally, the average value of each eigenvalue is used as each component in the texture vector. Since the physical meaning and value range of each component are different, they need to be internally normalized so that each component has the same weight. The model method matches the distribution of texture primitives with a certain mathematical model and analyzes the texture model with

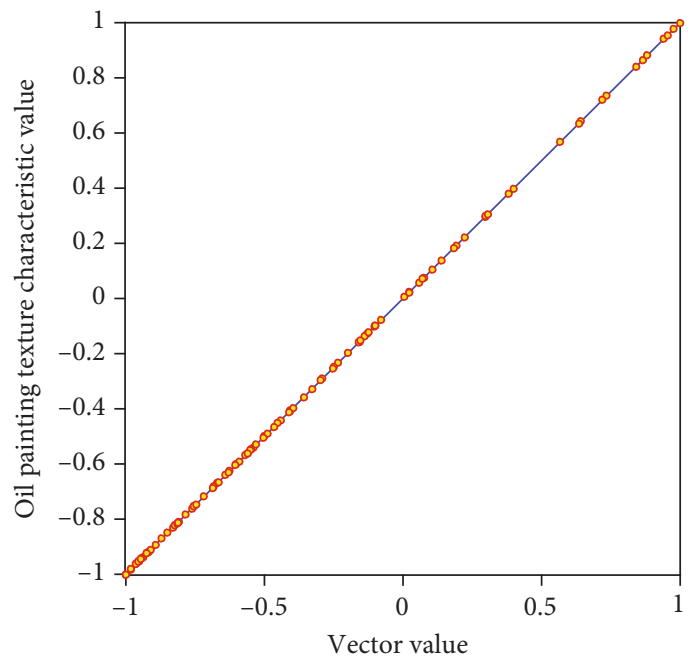

Figure 3: Fitting of the eigenvalues of oil painting texture with different vectors.

corresponding methods in theories such as statistics and signal analysis to obtain texture features. First, the mature oil painting model is used to determine the analytical model of texture oil painting, and then, the analytical model of each texture is represented by a texture feature parameter set. This set of texture feature parameters determines the texture feature of the texture model. Therefore, accurately estimating the feature parameter set of the model is the key to model-based texture analysis. Among them, the random field model has a low ability to describe regular and nonuniform textures. The texture image is regarded as a twodimensional signal, and the two-dimensional discrete 


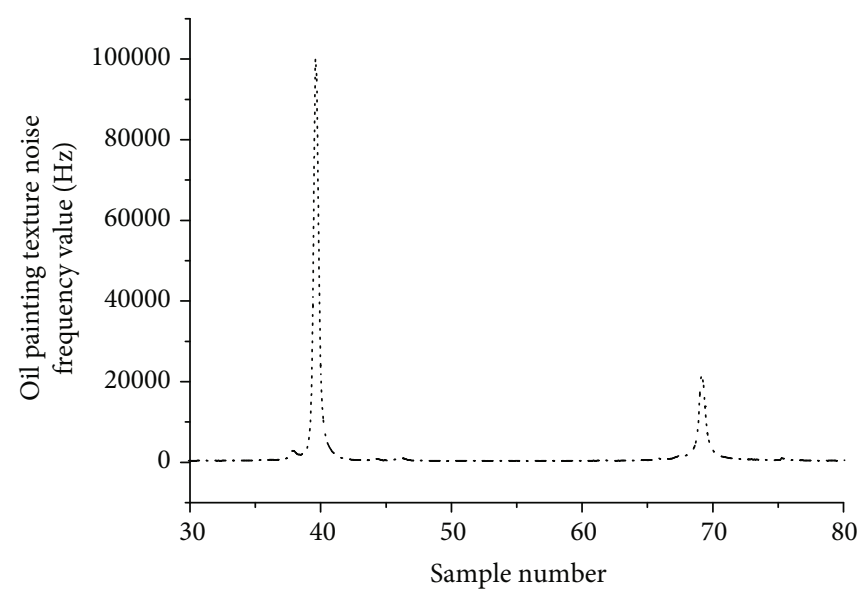

FIGURE 4: Numerical smoothing curve of oil painting texture noise frequency.

wavelet transform is used to process the texture image. The image can be decomposed in the frequency domain into low-frequency subbands (the basic structure of texture) and high-frequency subbands (texture details) in several directions, and then, the features of each subband can be extracted to form a feature vector. Generally, a matrix is first constructed according to the direction and distance between the oil picture elements, and then, meaningful statistical features are extracted from the matrix to describe the texture. Figure 4 shows the smoothing curve of oil painting texture noise frequency value.

During the movement of the window, most of the gray values remain unchanged; only the newly added column changes. If the value already exists, add the probabilities; if the value does not exist, add a new point to the linked list. Because texture is a representation of the relationship between the geometric positions of adjacent pixels or the gray levels of adjacent areas, the gray-level correlation of a pair of pixels in the same position relationship can be calculated by using a certain conditional probability of this pair of pixels.

\section{Application and Analysis of the Self-Adaptive Extraction Model of Oil Painting Texture Features Based on the Reaction- Diffusion Equation}

4.1. Preprocessing of Oil Painting Texture Data. Each classified oil painting also undergoes the oil painting preprocessing and texture feature extraction module to generate a test sample file. Each record in the file contains only 8 texture feature values without a category number. The test sample file is input into the multiclass SVM classification module. The multiclass SVM classification module uses the existing classification model to judge the type of the test sample and this document information using the voting method and returns the judged type number to the user. The collection time interval of each picture in the oil painting sequence shown is 0.1 second, and the iteration period is 1 second. It can be seen that in the process of oil painting evolution,
TABLE 2: Gray-scale attributes of oil painting texture.

\begin{tabular}{lcccc}
\hline $\begin{array}{l}\text { Serial } \\
\text { number }\end{array}$ & $\begin{array}{c}\text { Recognition } \\
\text { rate }(\%)\end{array}$ & $\begin{array}{c}\text { Noisy } \\
\text { image }\end{array}$ & $\begin{array}{c}\text { Median } \\
\text { filter }\end{array}$ & $\begin{array}{c}\text { Gaussian } \\
\text { filtering }\end{array}$ \\
\hline 1 & 88.21 & 0.47 & 25.07 & 24.97 \\
2 & 78.97 & 0.36 & 23.88 & 24.11 \\
3 & 82.43 & 0.29 & 23.21 & 25.61 \\
4 & 88.62 & 0.42 & 20.31 & 26.74 \\
\hline
\end{tabular}

the gray-scale conversion, and positive and negative mode flip of oil paintings have been realized. Moreover, the edge enhancement phenomenon has also appeared in the process of flipping the positive and negative modes of the image. Table 2 shows the gray-scale attributes of oil painting texture. A certain correlation between two gray-scale pixel values separated by a certain distance in a certain direction in an image is helpful to explain the information of the direction and amplitude change of the image gray scale. It can be seen that while the gray value of each point oscillates in the microscopic view, it also presents a variety of oil painting processing results that the entire oil painting evolves over time in the macroscopic view.

This experiment uses ground stationary target SAR data collected by the US MSTAR program. The size of the original oil painting is $128 \times 128$. The maximum value of the gray value gradient before and after filtering in the same window, $m$, represents the number of windows. In the case of relatively serious coherent speckles, due to the noise filtering, the noise filtering is more thorough and the edge protection index is relatively low, and the edge protection index of noisy oil paintings is high. Therefore, only the partial differential method which is better for noise filtering is compared with the method in this paper.

Figure 5 shows the histogram of the smoothing speed ratio of oil painting texture noise. It can be seen that the computational advantages of the statistical gray moment linked list method are obvious. When the gray level is 25 , the error is reduced by about $10 \%$, when the gray level is 44 , the error is reduced by about $8.7 \%$, and when the gray level is 66 , the error is reduced by about $4.7 \%$. It can be seen that the higher the gray level is, the more obvious the 


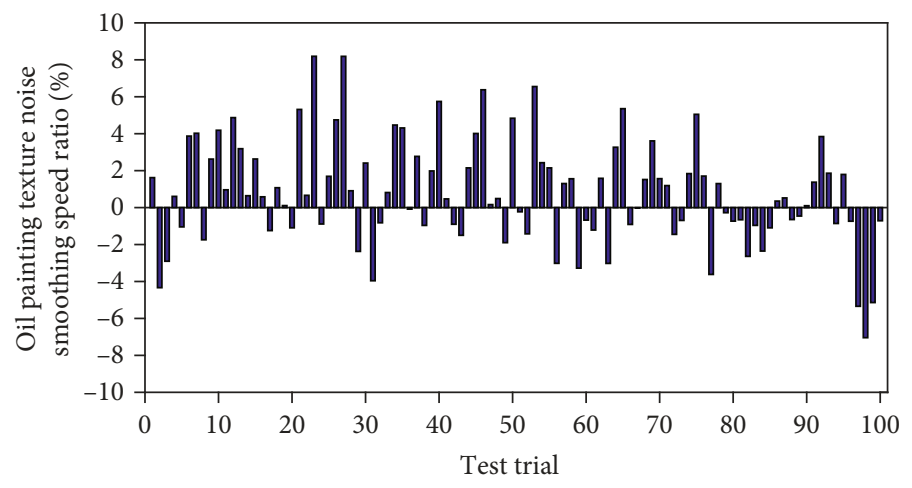

FIgURE 5: Oil painting texture noise smoothing speed ratio histogram.

advantages of the statistical gray moment linked list method are. Based on the above theoretical and experimental results, it can be seen that any single method has its own limitations. The thermal reaction diffusion equation and partial differential filtering can effectively smooth the noise but at the same time make the target fuzzy to a certain extent. Diffusion vibration filtering diffusion enhances the target, but the noise is also enhanced at the same time. The filtering effect is relatively good, but there are still many noise points after filtering, and the target is also blurred. Therefore, the statistical gray moment linked list method is suitable for texture feature extraction in oil painting classification. Because the method in this paper treats the target and noise separately, it not only fully suppresses the background noise, and the effect of strengthening the target is obvious. It shows that the composite method overcomes the problem that a single method deals with only one aspect, and can achieve the purpose of enhancing the target and suppressing background noise.

4.2. Adaptive Model Simulation. First, we select some oil paintings in the digital media resource library to participate in the training through the user interface, and mark the category number for each training oil painting. Each training oil painting is loaded with oil painting information through the oil painting preprocessing module, and the oil painting information is converted into a gray-scale image. The gray image data enters the texture feature extraction module. According to the algorithm and the statistical gray moment linked list algorithm, the gray level cooccurrence matrix, the sum difference statistical linked list, and the gray-scale matrix linked list are generated, and then, 8 typical texture feature values are calculated to obtain. We put the texture feature vectors of all the training oil paintings into the training sample file, and each record contains the oil painting category number and 8 texture feature values. The training sample files are input into the multiclass SVM training module, and the multiclass SVM training module uses the SMO training algorithm to analyze the file information and obtain multiple discriminant functions, namely, the classification model. Figure 6 shows a step diagram of texture feature recognition rate. The classification model is saved in the database. The image subimages are very similar, but the

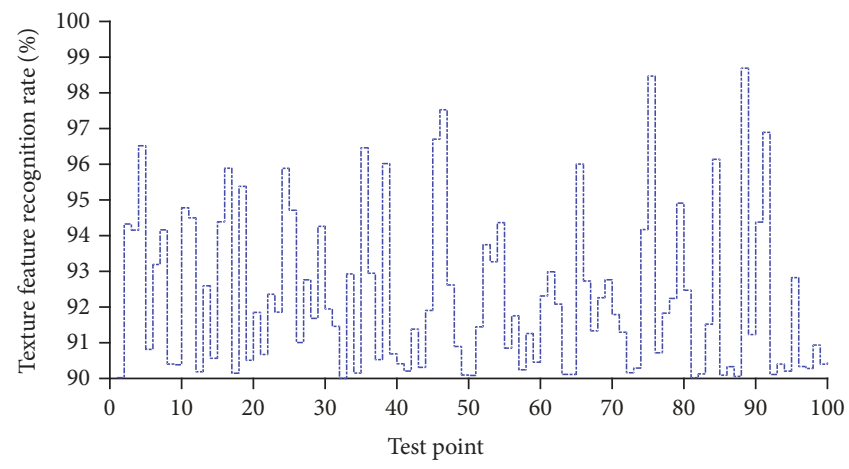

FIGURE 6: Ladder diagram of texture feature recognition rate.

training subimages and the test subimages are very different in brightness, which is an important reason for their judgment errors. When testing the category of oil paintings, we can choose to upload local oil paintings through the user interface or select oil paintings in the digital media resource library for category testing.

It can be seen that the edge of the target in the oil painting has been highlighted during the evolution process, which separates the target from the surrounding environment. Therefore, we can use this feature to detect and locate the target in the oil painting. The edge detection of oil painting by simulating the reaction medium is essentially different from the edge detection method in the classic earthworm image processing. In the classic method, edge detection often obtains the boundary information of the oil painting through filtering operations, while in this method, the edge detection result appears in the oil painting evolution cycle, and manual intervention is required to obtain the desired result. Compared with the serial algorithm running on the $\mathrm{CPU}$, the parallel oil painting interpolation algorithm implemented by CUDA running on the GPU has significantly improved the operation speed. At the same time, because the oil painting interpolation algorithm based on the PAR model has good separability and fastness, it is very suitable for the parallel architecture of GPU.

4.3. Example Application and Analysis. The experimental environment of this article: CPU is Intel Quad Core i7920; the main frequency is $2.66 \mathrm{GHz}$. The GPU of the graphics 


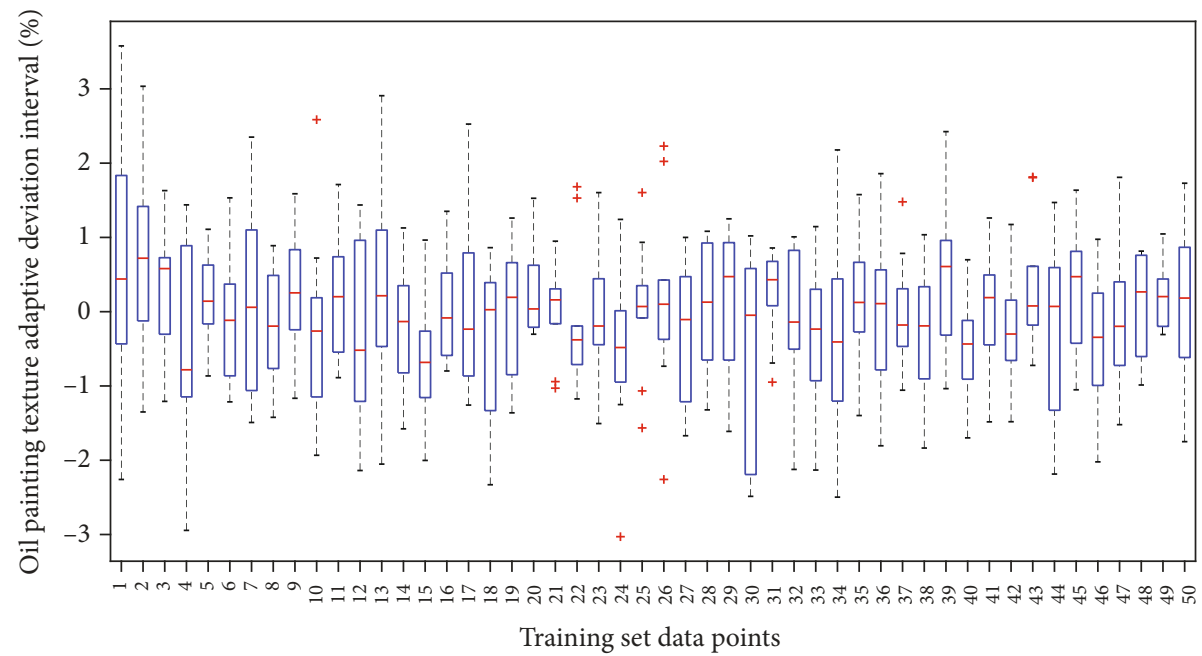

FIgURE 7: The statistical distribution of oil painting texture adaptive deviation error.

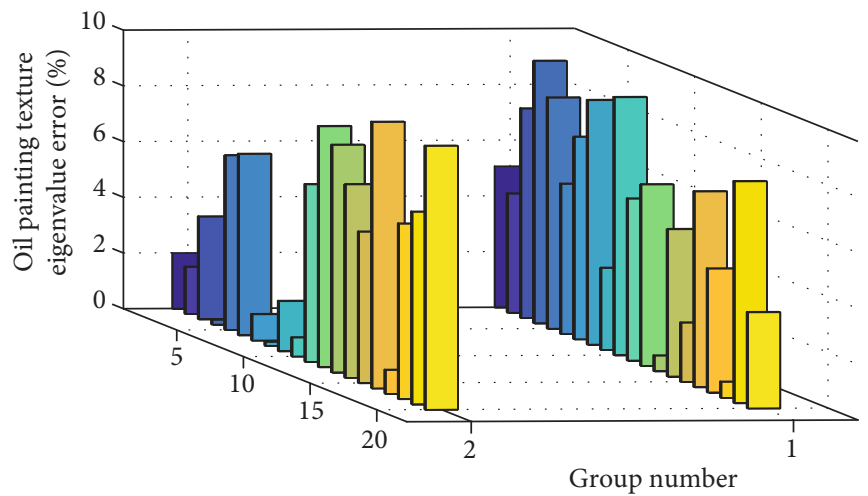

FIGURE 8: Three-dimensional histogram distribution of oil painting texture eigenvalue errors.

card is NVIDIA GTX285, the core frequency is $648 \mathrm{MHz}$, the video memory size is $1 \mathrm{G}$, the system memory is $2 \mathrm{G}$, and the operating system is Ubuntu 9.04. The algorithm implementation in this paper is based on the 2.3 version of CUDA SDK. In order to ensure the fairness of the experiment, the experimental data of experiment is oil painting. The computational performance of the program will not be much improved. The application of domain decomposition method can better solve this problem. First, the calculated rectangular area can be divided into subregions, and each processor can calculate a subregion. In order to comprehensively discuss the adaptability and popularization of the algorithm, we cut out more representative parts, including the low-frequency area and the high-frequency area, the original image size of the experiment intercepted is $100 \times$ 100 , and the nearest neighbor interpolation method, bilinear interpolation method, bicubic linear interpolation method, and cubic convolution interpolation method are used, respectively.

First, a triangular coarse mesh with a mesh number of 241 was established on the solution area. To solve on this mesh, we intercepted the finite element numerical solutions at different times. The mesh is uniformly refined, and a fine mesh with a unit number of 2034 is obtained. We can see that the position of the singularity of the solution is slowly moving with the change of time, and the adaptive mesh also changes with the change of the position of the singularity. Figure 7 shows the statistical distribution of the oil painting texture adaptive deviation error. Analyzing the calculation results at different times, it can be seen that with the increase of time, the number of grids is basically stable, and the error value fluctuates around 0.007 , which is obviously better than the results calculated on a consistent grid. The problem was also solved by finite element on the mesh, and we obtained the numerical solution of the finite element on the fine mesh at different times. We can see that the adaptive grid has been refined in places where the solution fluctuates sharply, and where the solution changes slowly, the adaptive grid method has performed a good simulation of the change of the solution. Analysis of the calculation results shows that with the increase of time, the number of grids is increasing, but the error is basically stable at around 0.125 , which is obviously better than the results calculated on a consistent grid, which proves that the algorithm in this paper is effective.

Figure 8 shows the three-dimensional histogram distribution of oil painting texture feature value errors. The experimental results show that the PAR model-based oil painting interpolation algorithm used in the article is much better 
than the nearest neighbor method and bilinear interpolation method in both the high-frequency region and the lowfrequency region and is much better than the bicubic linear interpolation method and cubic convolution interpolation method. The experimental results show that the edges of the method used in the article are more distinct and the visual quality is higher. Due to the interference of strong noise, our algorithm is not obvious in the interpolation effect of low-information parts such as fields. However, in places where the amount of information is relatively high, such as field ridges, it is obvious that our interpolation results are ideal. All threads can call and change the value of this array, and the variable is private because of the parameter added in front. Because the value is different in each thread, the data transfer task is completed by these threads. In order to further illustrate the effect of interpolation, using the proposed denoising algorithm to denoise the oil painting after dimensionality reduction, and then perform interpolation, it can be clearly seen that the result of the interpolation after denoising is obviously far better than other interpolation algorithms in edges and fine texture.

\section{Conclusion}

By analyzing the segmentation results of the unbounded active contour level set segmentation algorithm for oil paintings with different smoothness, we propose a new fast oil painting segmentation algorithm. The new algorithm removes the regular term of the original model and discrete functional energy on the gray level set. For calculation, the calculation time is very short, and the segmentation result is similar to the original model. Combining the characteristics and ideas of several types of methods, this paper proposes an improved algorithm-statistical gray moment linked list method. It can be seen in theory that the statistical gray moment linked list method solves the problem of large storage space requirements. Combining the improved graylevel cooccurrence matrix algorithm and the multiclass support vector machine classification model, the texture feature extraction, training, and classification of the image are carried out. Through the implementation of the traditional gray-level cooccurrence matrix algorithm and the improved algorithm and the experiments of extracting texture features on three groups of oil paintings, it is proved that the statistical gray moment linked list method takes much less time to calculate 8 typical texture feature values than the traditional ones. For the time required for the gray-level cooccurrence matrix algorithm, this article starts from the typical reaction diffusion system reaction, analyzes its kinetic characteristics and parameter value rules, simulates its kinetic behavior, and then discusses the special type of reaction diffusion equation-nonlinear expansion reaction diffusion equation and its specific application in the field of oil painting processing by using the reaction diffusion system wave.

\section{Data Availability}

The data used to support the findings of this study are available from the corresponding author upon request.

\section{Conflicts of Interest}

The author declares that there are no known competing financial interests or personal relationships that could have appeared to influence the work reported in this paper.

\section{Acknowledgments}

This work was supported by Shanxi Federation of Social Sciences Key project from 2021 to 2022: "Research on digital communication and promotion of Shanxi Guangling paper cutting" (No. SSKLZDKT 2021113).

\section{References}

[1] H. Yang, H. Li, and Y. Duan, "Adaptive trainable non-linear reaction diffusion for Rician noise removal," IET Image Processing, vol. 14, no. 14, pp. 3547-3561, 2020.

[2] J. Yu, J. Ye, and S. Zhou, "Reaction-diffusion system with additional source term applied to image restoration," International Journal of Computer Applications, vol. 147, no. 2, pp. 18-23, 2016.

[3] S. Ito, K. Uchizono, and R. Morita, "Extraction of moving areas in random-dot animation with parallel computation," Cybernetics and Systems, vol. 51, no. 5, pp. 561-578, 2020.

[4] M. Ushida, A. Schmid, T. Asai, K. Ishimura, and M. Motomura, "Motion vector estimation of textureless objects exploiting reaction-diffusion cellular automata," International Journal of Unconventional Computing, vol. 12, no. 23, pp. 169-187, 2016.

[5] M. Zirhem and N. E. Alaa, "Existence and uniqueness of an entropy solution for a nonlinear reaction- diffusion system applied to texture analysis," Journal of Mathematical Analysis and Applications, vol. 484, no. 1, article 123719, 2020.

[6] A. Hadri, H. Khalfi, A. Laghrib, and M. Nachaoui, "An improved spatially controlled reaction-diffusion equation with a non-linear second order operator for image super-resolution," Nonlinear Analysis: Real World Applications, vol. 62, article 103352, 2021.

[7] A. Atlas, M. Bendahmane, F. Karami, D. Meskine, and O. Oubbih, "A nonlinear fractional reaction-diffusion system applied to image denoising and decomposition," Discrete \& Continuous Dynamical Systems-B, vol. 26, no. 9, p. 4963, 2021.

[8] Y. Chen and T. Pock, "Trainable nonlinear reaction diffusion: a flexible framework for fast and effective image restoration," IEEE Transactions on Pattern Analysis and Machine Intelligence, vol. 39, no. 6, pp. 1256-1272, 2017.

[9] M. Chang, Q. Li, H. Feng, and Z. Xu, "Spatial-adaptive network for single image denoising," Computer Vision, vol. 12375, pp. 171-187, 2020.

[10] Y. Xing, J. Yu, F. Zhang, and Y. Gong, "Image denoising algorithm based on local adaptive nonlinear response diffusion," Materials Science and Engineering, vol. 790, no. 1, p. 12103, 2020.

[11] Q. Zhang, S. Song, Y. Xiao, S. Chen, J. Shi, and H. Zheng, "Dual-mode artificially-intelligent diagnosis of breast tumours in shear-wave elastography and B-mode ultrasound using deep polynomial networks," Medical Engineering \& Physics, vol. 64, pp. 1-6, 2019.

[12] W. Yao, Z. Guo, J. Sun, B. Wu, and H. Gao, "Multiplicative noise removal for texture images based on adaptive anisotropic 
fractional diffusion equations," SIAM Journal on Imaging Sciences, vol. 12, no. 2, pp. 839-873, 2019.

[13] Z. Zhang, Y. M. Xie, Q. Li, and S. Zhou, "A reaction-diffusion based level set method for image segmentation in three dimensions," Engineering Applications of Artificial Intelligence, vol. 96, p. 103998, 2020.

[14] M. T. Chi, W. C. Liu, and S. H. Hsu, "Image stylization using anisotropic reaction diffusion," The Visual Computer, vol. 32, no. 12, pp. 1549-1561, 2016.

[15] W. Wen, C. He, Y. Zhang, and Z. Fang, "A novel method for image segmentation using reaction-diffusion model," Multidimensional Systems and Signal Processing, vol. 28, no. 2, pp. 657-677, 2017.

[16] X. Zhao, K. Huang, X. Wang et al., "Reaction-diffusion equation based image restoration," Applied Mathematics and Computation, vol. 338, pp. 588-606, 2018.

[17] A. Foadaddini, S. A. Zolfaghari, H. Mahmoodi Darian, and H. Saadatfar, "An efficient GPU-based fractional-step domain decomposition scheme for the reaction-diffusion equation," Computational and Applied Mathematics, vol. 39, no. 4, pp. 29-35, 2020.

[18] K. Shi, D. Zhang, Z. Guo, and B. Wu, "A linear reactiondiffusion system with interior degeneration for color image compression," SIAM Journal on Imaging Sciences, vol. 11, no. 1, pp. 442-472, 2018.

[19] A. Fofonjka and M. C. Milinkovitch, "Reaction-diffusion in a growing 3D domain of skin scales generates a discrete cellular automaton," Nature Communications, vol. 12, no. 1, pp. 10$13,2021$.

[20] N. Henscheid, "Generating patient-specific virtual tumor populations with reaction-diffusion models and molecular imaging data," Mathematical Biosciences and Engineering, vol. 17, no. 6, pp. 6531-6556, 2020.

[21] F. Karami, D. Meskine, and K. Sadik, "A new nonlocal model for the restoration of textured images," Journal of Applied Analysis \& Computation, vol. 9, no. 6, pp. 2070-2095, 2019.

[22] A. Onan, "A fuzzy-rough nearest neighbor classifier combined with consistency-based subset evaluation and instance selection for automated diagnosis of breast cancer," Expert Systems with Applications, vol. 42, no. 20, pp. 6844-6852, 2015.

[23] J. Chen and G. Tang, "A feature selection model to filter periodic variable stars with data-sensitive light-variable characteristics," Journal of Signal Processing Systems, vol. 93, no. 7, pp. 733-744, 2021. 